\title{
Maximin and Maximal Solutions for Linear Programming Problems with Possibilistic Uncertainty
}

\author{
Erik Quaeghebeur, Nathan Huntley, \\ Keivan Shariatmadar, Gert de Cooman \\ Ghent University, SYSTeMS Research Group \\ Technologiepark-Zwijnaarde 914, 9052 Zwijnaarde, Belgium
}

\begin{abstract}
We consider linear programming problems with uncertain constraint coefficients described by intervals or, more generally, possibility distributions. The uncertainty is given a behavioral interpretation using coherent lower previsions from the theory of imprecise probabilities. We give a meaning to the linear programming problems by reformulating them as decision problems under such imprecise-probabilistic uncertainty. We provide expressions for and illustrations of the maximin and maximal solutions of these decision problems and present computational approaches for dealing with them.
\end{abstract}

Keywords: linear program, interval uncertainty, vacuous lower prevision, possibility distribution, coherent lower prevision, imprecise probabilities, decision making, maximinity, maximality.

\section{Introduction}

Linear programming problems thank their importance both to the great variety of optimization questions that can be modeled by them and to the existence of computationally efficient algorithms for solving them [2. A linear programming problem can be expressed in the following full (left) and compact (right) forms:

$$
\begin{aligned}
& \text { maximize } \sum_{k=1}^{n} c_{k} x_{k} \quad \text { maximize } c^{T} x \\
& \text { subject to } \forall_{\ell=1}^{m}\left(\sum_{k=1}^{n} a_{\ell k} x_{k} \leq b_{\ell}\right), \equiv \text { subject to } a x \leq b \text {, } \\
& \forall_{k=1}^{n}\left(x_{k} \geq 0\right) \quad x \geq 0
\end{aligned}
$$

where $x$ in $\mathbb{R}^{n}$ is the optimization vector of dimension $n \in \mathbb{N}, a$ in $\mathbb{R}^{m \times n}$ and $b$ in $\mathbb{R}^{m}$ are the constraint coefficient matrix and (column) vector with $m \in \mathbb{N}$ the number of nontrivial constraints, and $c$ in $\mathbb{R}^{n}$ is the objective function coefficient vector $-c^{T}$ is its transpose. (The lower case matrix notation is justified below.)

We are interested in linear programming problems in which there is uncertainty in some or all of the constraints. Being able to treat them allows us to more realistically deal with real-life operations research problems [8, Section 5].

If we are uncertain about the value of a particular constraint coefficient, we represent it by an upper case letter, e.g., $A_{\ell k}$ or $B_{\ell}$, and similarly $A$ and $B$ if this 
matrix and vector have one or more uncertain components. To these (matrices or vectors of) uncertain coefficients we must associate a model to formalize the uncertainty. These problems can then be expressed as

$$
\begin{aligned}
\text { maximize } & c^{T} x \\
\text { subject to } & A x \leq B, x \geq 0 \\
\text { with } & \text { given uncertainty model for }(A, B)
\end{aligned}
$$

This uncertainty means that, for a given choice of $x$, it may be uncertain whether $x$ satisfies the constraints or not, and therefore it is not clear what it means to 'maximize' $c^{T} x$ in such a problem.

Our approach is to transform the problem into a decision problem (Section 2 , in which a fixed penalty is received if any of the constraints are broken [7. Since this is a reformulation as a decision problem, it can then in principle be solved using a suitable optimality criterion for the uncertainty models being used, for instance maximizing expected utility when probability distributions are used, as we do in Section 3 to introduce the basic ideas. In Section 4 , we show how these ideas are generalized to all uncertainty models that can be seen as coherent lower or upper previsions [1] and introduce maximinity and maximality, the two chosen amongst many compatible optimality criteria. We focus on two specific types of uncertainty models: intervals - vacuous lower probabilities - in Section 5 and, more generally, possibility distributions - maxitive upper probabilities - in Section 6. A simplifying assumption we make in this paper is that the uncertainty models for the various uncertain constraint coefficients are independent, the formal meaning of which we will make precise for each type.

We were surprised at how different this approach is from those usually found in the literature - we only have space for a fleeting overview. First the probabilistic case: Dantzig [4] looked at the problem as a staged one: first choose $x$, observe $(A, B)$, and then 'repair' broken constraints. Charnes \& Cooper [3] proposed to solve the problem under the added 'chance constraint' that the probability of failing a constraint is below some level. Our solution for the interval case with maximinity, although arrived at differently, essentially reduces to the approach of Soyster [9]; further results in this vein can be found in the domains of inexact and robust optimization [5]1. The possibility distribution case has been analyzed from a very wide range of angles by the fuzzy sets community - all nevertheless differing from ours; the approach of Jamison \& Lodwick [6] is one to mention, because they also pursue a penalty idea, but use a different optimality criterion.

To illustrate our methods, we introduce a running example, in which only one constraint coefficient is uncertain (left: full form, right: shorthand form):

$$
\begin{aligned}
\operatorname{maximize} & 2 x_{1}+3 x_{2} \\
\text { subject to } & 1 x_{1}+3 x_{2} \leq 2, \\
& 1 x_{1}+1 x_{2} \leq B_{2}, \quad \equiv \quad \begin{aligned}
& \\
&- 3 x_{1}-3 x_{2} \leq-1, \\
& x_{1} \geq 0, x_{2} \geq 0
\end{aligned} \text { subject to } \quad x \triangleleft B_{2}
\end{aligned}
$$

For reference, we first show, in Figure 1 the usual linear programming problem, i.e., for some particular precisely known $B_{2}$, and its solution. 


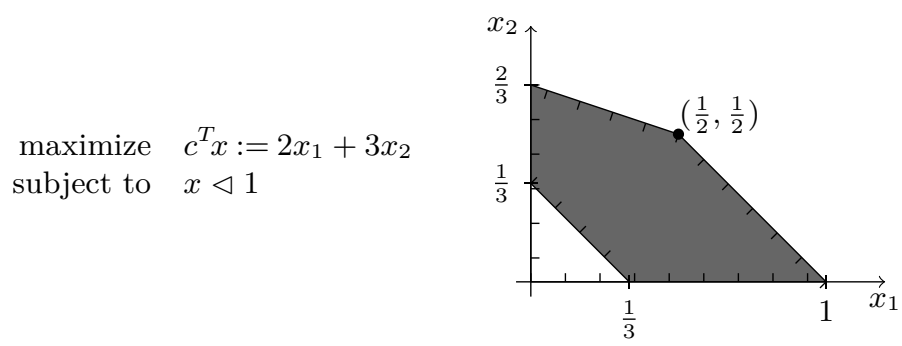

Figure 1. Example linear programming problem: the constraints are drawn using hairy lines; the feasible set is shaded dark gray; the convex solution set of optimization vectors attaining the maximal value $5 / 2$ is a singleton in this case, represented as a black dot.

\section{Reformulation as a Decision Problem}

Now we describe the decision problem that we use in the case of uncertainty. For each optimization vector $x$ and each possible realization $(a, b)$ of $(A, B)$, we associate a utility $c^{T} x$, unless $x$ fails a constraint, in which case the utility becomes a penalty value $L$. This value $L$ should be chosen so it is strictly worse to fail the constraints than to satisfy them, and so $L$ should be strictly less than $c^{T} x$ for any $x$ that is feasible for some possible realization $(a, b)$.

We use $A x \leq B$ as a shorthand for the event $\left\{(a, b) \in \mathbb{R}^{m \times n} \times \mathbb{R}^{m}: a x \leq b\right\}$ and so the corresponding indicator function $I_{A x \leq B}$ is 1 on this event and 0 elsewhere; idem for $A x \not \leq B$. Then the gain or utility function associated to $x$ is

$$
G_{x}:=c^{T} x I_{A x \leq B}+L I_{A x \nless B}=L+\left(c^{T} x-L\right) I_{A x \leq B} .
$$

Given such a utility function and an uncertainty model for $(A, B)$, we can use a suitable optimality criterion to determine the optimal choices of $x$.

As can be gleaned from the formulation of our running example and Figure 1 independent of the uncertainty about $B_{2}$, any feasible optimization vector $x$ will have a value of at least $2 / 3$, as achieved in $(1 / 3,0)$. Therefore we are allowed the choice $L=0$, which simplifies our discussion of the running example.

\section{Probability Mass Functions}

A simple probabilistic case serves as a good introduction to the main ideas of our method. Assume the uncertainty about the independent scalar uncertain variables is expressed using probability mass functions and let $p$ be the corresponding product probability mass function for $(A, B)$ with Cartesian product support $\mathcal{A} \times \mathcal{B}$. Let $P$ denote both the associated probability measure for subsets of $\mathcal{A} \times \mathcal{B}$ and linear prevision (expectation operator) for gambles (real-valued functions) on $\mathcal{A} \times \mathcal{B}$.

Utility functions are gambles. The expected utility of choosing an optimization vector $x \geq 0$ is

$$
P\left(G_{x}\right)=P\left(L+\left(c^{T} x-L\right) I_{A x \leq B}\right)=L+\left(c^{T} x-L\right) P(A x \leq B) .
$$


We give meaning to the linear program with uncertain constraints thus defined by saying that the solution must maximize expected utility:

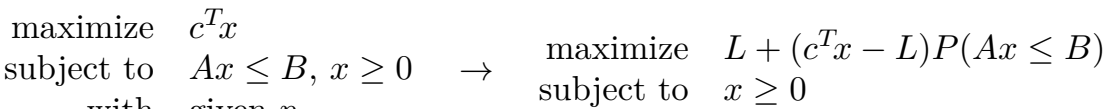

$$
\begin{aligned}
& \text { with given } p
\end{aligned}
$$

As the objective function is in general not affine in $x$, the resulting optimization problem is in general no longer a linear program. In general, it is also difficult to compute the probabilistic factor $P(A x \leq B)$, let alone find an expression for it. In Figure 2, we give a simple example that can nevertheless be solved by hand.

We use a conceptually useful generalization of the feasibility concept: an optimization vector $x$ is $P(A x \leq B)$-feasible; for correspondence with the standard case, 0 -feasible vectors are called infeasible and 1 -feasible vectors feasible.

$$
\begin{aligned}
\text { maximize } & c^{T} x:=2 x_{1}+3 x_{2} \\
\text { subject to } & x \triangleleft B_{2} \\
\text { with } & p_{B_{2}}(b)= \begin{cases}3 / 5, & b=1, \\
1 / 5, & b \in\{2 / 3,4 / 3\}, \\
0, & \text { elsewhere }\end{cases} \\
& \downarrow \text { MEU }(L=0) \\
\text { maximize } & P\left(B_{2} \geq b\right)\left(\begin{array}{cl}
\text { maximize } & c^{T} x \\
\text { subject to } & x \triangleleft b
\end{array}\right) \\
\text { subject to } & b \in\{2 / 3,1,4 / 3\}
\end{aligned}
$$

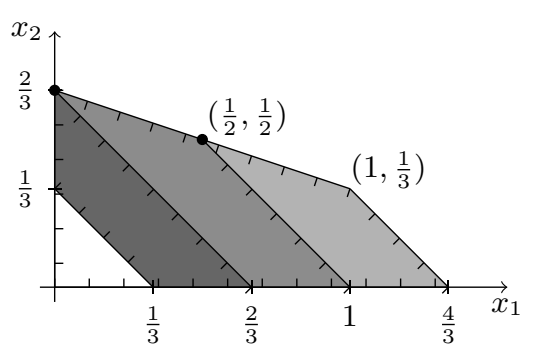

Figure 2. Example linear programming problem with probabilistic uncertainty: the feasible set is shaded dark gray, the $4 / 5$-feasible set gray, and the $1 / 5$-feasible set light gray; because the set of optimization vectors is partitioned according to probability value, the maximization can be done in a nested manner; the solution set of optimization vectors attaining the maximal expected value 2 is given using black dots.

\section{Generalizing the Probabilistic Case}

Coherent lower and upper previsions. A wide range of uncertainty models can be represented using coherent lower and upper previsions from the theory of imprecise probabilities [11]. Like linear previsions, these are expectation operators, but whereas linear previsions can be interpreted as specifying fair prices for buying and selling gambles, coherent lower and upper previsions can be respectively interpreted as specifying supremum acceptable buying prices and infimum acceptable selling prices. They are conjugate operators; given a coherent lower prevision $\underline{P}$, the conjugate coherent upper prevision is given by $\bar{P}(f)=-\underline{P}(-f)$ for every gamble $f$.

Coherent lower and upper probabilities are derived concepts for which we use the same symbol: given an event $\mathcal{E}$, we have $\underline{P}(\mathcal{E})=\underline{P}\left(I_{\mathcal{E}}\right)$ and $\bar{P}(\mathcal{E})=\bar{P}\left(I_{\mathcal{E}}\right)$. As 
before, we generalize the feasibility concept: an optimization vector $x$ is called inner $\underline{P}(A x \leq B)$-feasible and outer $\bar{P}(A x \leq B)$-feasible; for correspondence with the standard case, outer 0 -feasible vectors are called infeasible and inner 1 -feasible points feasible.

Optimality criteria. When using coherent lower and upper previsions it is no longer possible to maximize expected utility. Several generalizations have been proposed, of which we consider maximinity and maximality. Troffaes [10] gives a more detailed discussion of these and other optimality criteria.

Under the maximinity criterion, applied to our problem, those optimization vectors $x \geq 0$ are optimal that maximize the lower expected utility

$$
\underline{P}\left(G_{x}\right)=\underline{P}\left(L+\left(c^{T} x-L\right) I_{A x \leq B}\right)=L+\left(c^{T} x-L\right) \underline{P}(A x \leq B) .
$$

Under the maximality criterion, those $x \geq 0$ are optimal that are undominated in comparisons with all other optimization vectors in the following sense:

$$
\inf _{z \in \mathbb{R}^{n}} \bar{P}\left(G_{x}-G_{z}\right)=\inf _{z \in \mathbb{R}^{n}} \bar{P}\left(\left(c^{T} x-L\right) I_{A x \leq B}-\left(c^{T} z-L\right) I_{A z \leq B}\right) \geq 0 .
$$

Additionally, we perform a further selection among maximin or maximal solutions by imposing dominance, pointwise comparisons of utility functions:

$$
\forall z \in \mathbb{R}^{n}: G_{z}=G_{x} \text { or } \max \left(G_{x}-G_{z}\right)>0 .
$$

Applied to our problem, pairwise comparisons between $x$ and $z$ differ qualitatively based on where $G_{x}$ and $G_{z}$ take a value larger than $L$ :

(i) If $A x \leq B \nsubseteq A z \leq B$, then $z$ does not dominate $x$ because $G_{x}$ and $G_{z}$ are incomparable.

(ii) If $A x \leq B=A z \leq B$, then $z$ dominates $x$ if $c^{T} x<c^{T} z$ and therefore $x$ must satisfy $c^{T} x \geq \max _{(A x \leq B)=(A z \leq B)} c^{T} z$; in particular, if $A z \leq B$ is empty for all $z$, no selection is made - we do not consider this trivial case further on.

(iii) If $A x \leq B \subset A z \leq B$, then $z$ dominates $x$ if $c^{T} x \leq c^{T} z$ and therefore $x$ must satisfy $c^{T} x>\max _{(A x \leq B) \subset(A z \leq B)} c^{T} z$; in particular, if $A z \leq B$ is nonempty, all those $x$ for which $A x \leq B$ is empty are weeded out.

In general, and also for the problems we consider in this paper, checking dominance in a computationally efficient way remains an open problem.

\section{Intervals}

Assume the uncertainty about the $A_{\ell k}$ and $B_{\ell}$ is expressed using real intervals $\left[\underline{a_{\ell k}}, \overline{a_{\ell k}}\right]$ and $\left[\underline{b_{\ell}}, \overline{b_{\ell}}\right]$. Independence is implemented by taking Cartesian products such as $\mathcal{A}:=[\underline{a}, \bar{a}]:=\times_{1 \leq k \leq n, 1 \leq \ell \leq m}\left[\underline{a_{\ell k}}, \overline{a_{\ell k}}\right]$ and $\mathcal{B}:=[\underline{b}, \bar{b}]:=\times_{1 \leq \ell \leq m}\left[\underline{b_{\ell}}, \overline{b_{\ell}}\right]$. Then we can model the uncertainty using joint vacuous coherent lower and upper previsions $\underline{P}$ and $\bar{P}$ defined for every gamble $f$ on $\mathcal{A} \times \mathcal{B}$ by

$$
\underline{P}(f):=\min _{(a, b) \in \mathcal{A} \times \mathcal{B}} f(a, b) \quad \text { and } \quad \bar{P}(f):=\max _{(a, b) \in \mathcal{A} \times \mathcal{B}} f(a, b) .
$$


Intervals \& Maximinity. In case the maximinity criterion is used, Equation (3) shows the quantity $\underline{P}(A x \leq B)$ is important. By Definition (6), it is $1-$ and $x$ is feasible -if and only if $x$ satisfies the constraints $a x \leq b$ for all $\underline{a} \leq a \leq \bar{a}$ and $\underline{b} \leq b \leq \bar{b}$; otherwise it is 0 . Because we may assume $x \geq 0$, componentwise, the inequality's left-hand side will be maximal and the right-hand side minimal for $a=\bar{a}$ and $b=\underline{b}$; so if the constraints are satisfied for these values, they are also satisfied for all the others: the feasible set is $\bar{a} X \leq \underline{b}:=\{x \geq 0: \bar{a} x \leq \underline{b}\}$.

For feasible $x$, Equation (3) shows that the lower prevision is equal to $c^{T} x-$ the penalty $L$ falls out of the equation. So if the feasible set is nonempty, the maximin solutions are the those that maximize this quantity:

$$
\begin{aligned}
& \text { maximize } c^{T} x \quad \text { maximize } c^{T} x \\
& \text { subject to } A x \leq B, x \geq 0 \quad \rightarrow \quad \text { subject to } \bar{a} x \leq \underline{b} \text {, } \\
& \text { with } \quad \underline{a} \leq A \leq \bar{a}, \underline{b} \leq B \leq \bar{b} \quad x \geq 0
\end{aligned}
$$

The resulting optimization problem is then again a linear program. This is illustrated in Figure 3 Dominance is automatically satisfied, because $A x \leq B$ is equal (to $\mathcal{A} \times \mathcal{B}$ ) for all feasible $x$.
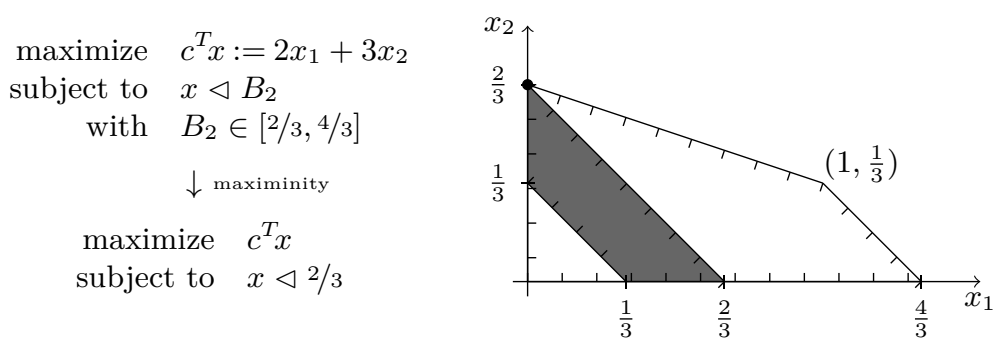

Figure 3. Example linear programming problem with interval uncertainty and maximinity: the feasible set is shaded dark gray; the convex solution set of optimization vectors attaining the maximal lower expected - i.e., maximin — value 2 is a singleton in this case, represented as a black dot.

If the feasible set is empty, all $x \geq 0$ are maximin solutions. But now dominance does come into play; e.g., Dominance (iii) weeds out everything outside of $\underline{a} X \leq \bar{b}$, which is the outer feasible set.

Intervals \& Maximality. In case the maximality criterion is used, Equation (4) shows the quantity $\bar{P}\left(G_{x}-G_{z}\right)$ is important. Definition (6) tells us that for optimization vectors $x$ and $z$ we have $\bar{P}\left(G_{x}-G_{z}\right) \geq 0$ if and only if there is a pair $(a, b)$ in $\mathcal{A} \times \mathcal{B}$ such that $G_{x}(a, b) \geq G_{z}(a, b)$.

In Dominance (iii), we stated that we only considered problems for which the outer feasible set $\underline{a} X \leq \bar{b}$ is nonempty. As seen above, Dominance (iii) tells us we can restrict attention to the outer feasible set when looking for maximal solutions. Now, outer feasible $x$ that satisfy dominance (5) also satisfy Equation (4), because the latter here reduces to $\min _{\underline{a} z \leq \bar{b}} \max \left(G_{x}-G_{z}\right) \geq 0$. 


$$
\begin{aligned}
\operatorname{maximize} & c^{T} x:=2 x_{1}+3 x_{2} \\
\text { subject to } & x \triangleleft B_{2} \\
\text { with } & B_{2} \in[2 / 3,4 / 3] \\
& \downarrow \text { maximality }
\end{aligned}
$$

maximize $\quad 0 x_{1}+0 x_{2}$

subject to $\quad x \triangleleft 4 / 3$

$$
\begin{aligned}
& c^{T} x \geq \max _{z \triangleleft 2 / 3} c^{T} z, \\
& c^{T} x \geq \max _{1 z_{1}+1 z_{2} \leq 1 x_{1}+1 x_{2}} c^{T} z \text { (dominance) }
\end{aligned}
$$

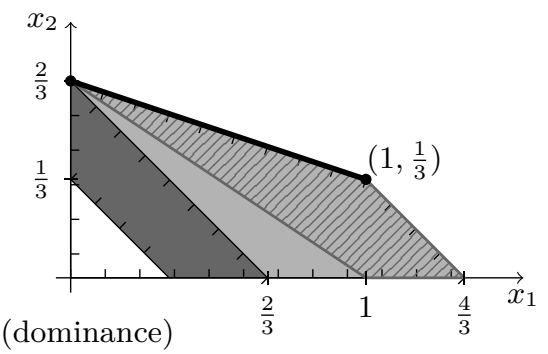

Figure 4. Example linear programming problem with interval uncertainty and maximality: the feasible set is shaded dark gray and the 1-outer feasible set light gray; the maximin solution corresponds to the leftmost black dot; the convex solution set of outer feasible points with an upper expected value greater than or equal to the maximin value is given by the hatched area; the maximal solutions satisfying dominance- given by the thick black line - are the optimization vectors $x$ corresponding to the gambles $\left(2 x_{1}+3 x_{2}\right) I_{x \triangleleft B_{2}}=\left(2 x_{1}+3 x_{2}\right) I_{1 x_{1}+1 x_{2} \leq B_{2}}$ undominated within this hatched set.

If moreover the feasible set $\bar{a} Z \leq \underline{b}$ is nonempty, then for outer feasible $x-$ with $\bar{P}(A x \leq B)=1$ - we have by sub-additivity of $\bar{P}$ [1] and conjugacy that

$$
\min _{\bar{a} z \leq \underline{b}} \bar{P}\left(G_{x}-G_{z}\right) \leq \bar{P}\left(G_{x}\right)-\max _{\bar{a} z \leq \underline{b}} \underline{P}\left(G_{z}\right)=c^{T} x-\max _{\bar{a} z \leq \underline{b}} c^{T} z .
$$

So no $x$ with $c^{T} x$ smaller than the value $\max _{\bar{a} z \leq b} c^{T} z$ of a maximin solution can be maximal. Actually, this is already implied by Dominance (ii) and (iii), but this criterion can be checked efficiently.

So $x$ is maximal if and only if it satisfies dominance and $a x \leq \bar{b}$, so maximality for intervals can be expressed as

$$
\begin{aligned}
& \text { maximize } c^{T} x \quad \text { maximize } 0^{T} x \\
& \text { subject to } A x \leq B, x \geq 0 \rightarrow \text { subject to } \quad \underline{a x} \leq \bar{b}, x \geq 0 \text {, } \\
& \text { with } \quad \underline{a} \leq A \leq \bar{a}, \quad \rightarrow \quad c^{T} x \geq \max _{\bar{a} z \leq b} c^{T} z, \\
& \underline{b} \leq B \leq \bar{b} \quad \text { dominance }
\end{aligned}
$$

The resulting optimization problem is a classical feasibility problem compounded with a dominance problem. This case is illustrated in Figure 4

\section{Possibility Distributions}

Assume that the uncertainty models for the $A_{\ell k}$ and $B_{\ell}$ are unimodal possibility distributions $\pi_{\ell k}$ and $\pi_{\ell}$. Independence is implemented with the usualleast complex - definition of joint possibility: $\pi_{A}(a):=\min _{1 \leq k \leq n, 1 \leq \ell \leq m} \pi_{\ell k}\left(a_{\ell k}\right)$, $\pi_{B}(b):=\min _{1 \leq \ell \leq m} \pi_{\ell}\left(b_{\ell}\right)$, and $\pi(a, b):=\min \left\{\pi_{A}(a), \pi_{B}(b)\right\}$. For any $D$ among the $A_{\ell k}$ and $B_{\ell}$, and $0 \leq t<1$, we define bounds $\underline{d}_{t}:=\inf \{d \in \mathbb{R}: \pi(d)>t\}$ and $\bar{d}_{t}:=\sup \{d \in \mathbb{R}: \pi(d)>t\}$. We write $\underline{a}_{t}, \bar{a}_{t}, \underline{b}_{t}$, and $\bar{b}_{t}$ for the matrices and vectors with respective components $\underline{a}_{\ell k_{t}}, \bar{a}_{\ell k}, \underline{b}_{t}$, and $\bar{b}_{\ell_{t}}$. Then $\mathcal{A} \times \mathcal{B}:=\left[\underline{a}_{0}, \bar{a}_{0}\right] \times\left[\underline{b}_{0}, \bar{b}_{0}\right]$ is (the closure of) the set of possible realizations of $(A, B)$. 
We can model the uncertainty using a joint upper probability $\bar{P}$ defined for every subset $\mathcal{E}$ of $\mathcal{A} \times \mathcal{B}$ by $\bar{P}(\mathcal{E}):=\sup _{(a, b) \in \mathcal{E}} \pi(a, b)$-lower probabilities follow by conjugacy and lower and upper previsions by Choquet integration [7].

Possibility Distributions \& Maximinity. By Equation (3), we seek to maximize $\underline{P}\left(G_{x}\right)=L+\left(c^{T} x-L\right) \underline{P}(A x \leq B)=L+\left(c^{T} x-L\right)(1-P(A x \not \leq B))$. With every $x$ there corresponds a unique value for $\bar{P}(A x \not \leq B)$, so this maximization can be done in a nested manner:

$$
\begin{aligned}
\max _{x \geq 0} \underline{P}\left(G_{x}\right) & =L+\max _{t \in[0,1]}(1-t) \max _{x \geq 0}\left\{c^{T} x-L: \bar{P}(A x \not \leq B)=t\right\} \\
& =L+\max _{t \in[0,1]}(1-t) \max _{x \geq 0}\left\{c^{T} x-L: \bar{P}(A x \not \leq B) \leq t\right\},
\end{aligned}
$$

where the second equality follows from the fact that for $\bar{P}(A x \not \leq B)=s<t$ with fixed $x$ and $t$ it holds that $(1-s)\left(c^{T} x-L\right) \geq(1-t)\left(c^{T} x-L\right)$.

Next we show that $\bar{P}(A x \nless B)=r:=\inf \left\{0 \leq \tau<1: \bar{a}_{\tau} x \leq \underline{b}_{\tau}\right\}$ : We have $a x \leq b$ for $a$ and $b$ such that $\pi(a, b)>r$, hence $\bar{P}(A x \leq B) \leq r$. For any $s<r$ we have $\bar{a}_{s} x \not \leq \underline{b}_{s}$, hence $\bar{P}(A x \not \leq B)=\sup _{(a, b) \in \mathcal{A} \times \mathcal{B}}\{\pi(a, b): a x \not \leq b\} \geq r$. It follows that $\bar{P}(A x \not \leq B) \leq t$ if and only if $\bar{a}_{t} x \leq \underline{b}_{t}$.

Hence the problem becomes

$$
\begin{aligned}
& \text { maximize } c^{T} x
\end{aligned}
$$

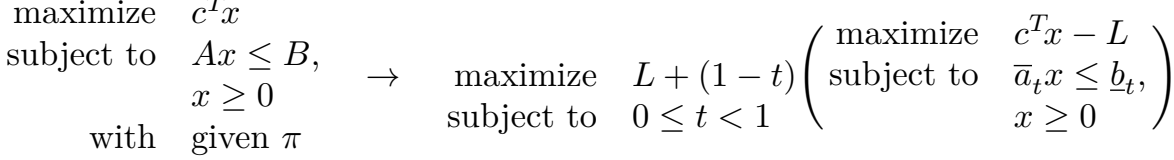

So we maximize over all $0 \leq t \leq 1$, where for any particular $t<1$, we just need to solve a single linear program. This maximization over $t$ can be done using, e.g., a bisection algorithm. Dominance is again automatically satisfied, now because $A x \leq B$ is equal (to $\bar{a}_{t} x \leq \underline{b}_{t}$ ) for all $(1-t)$ )-inner feasible $x$. This case is illustrated in Figure 5
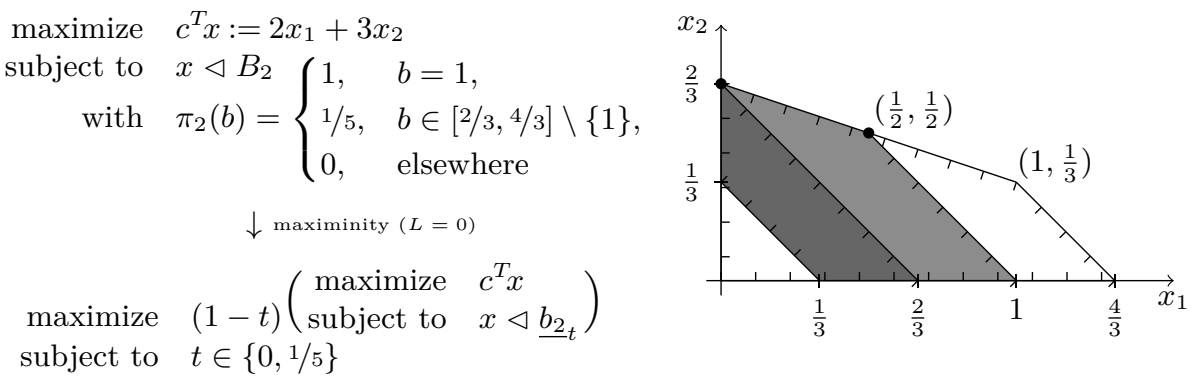

Figure 5. Example linear programming problem with possibilistic uncertainty and maximinity: the feasible set is shaded dark gray and the $2 / 3$-inner feasible set gray; the solution set of optimization vectors attaining the maximin value 2 is given using black dots. 
Possibility Distributions \& Maximality. We again start by focusing on the core of Equation (4), which can be written as [7]:

$$
\bar{P}\left(G_{x}-G_{z}\right)=\left\{\begin{aligned}
-\left(c^{T} z-L\right) & +\left(c^{T} x-c^{T} z\right) \bar{P}(A x \leq B) \\
& +\left(c^{T} z-L\right) \bar{P}(A x \leq B \vee A z \not \leq B) \\
& +\left(c^{T} z-L\right) \bar{P}(A x \leq B \wedge A z \not \leq B), \quad c^{T} x \geq c^{T} z, \\
-\left(c^{T} z-L\right) & -\left(c^{T} x-c^{T} z\right) \bar{P}(A z \not \leq B) \\
& +\left(c^{T} x-L\right) \bar{P}(A x \leq B \vee A z \not \leq B) \\
& +\left(c^{T} x-L\right) \bar{P}(A x \leq B \wedge A z \not \leq B), \quad c^{T} x \leq c^{T} z .
\end{aligned}\right.
$$

In this expression, only the factor $\bar{P}(A x \leq B \wedge A z \not \leq B)$ is hard to compute for given $x$ and $z$. However, to determine whether or not $z$ dominates $x$, we only need to find out whether $\bar{P}\left(G_{x}-G_{z}\right)<0$ or not, so we do not need to compute the factor's value, but only whether it is larger than some critical value that is a function of the (easily computable) rest of the expression. At the current state of our investigations, this comparison requires us to solve at most $m$ linear programs per $(x, z)$-pair.

We have not yet found a way to exploit this result to derive an explicit optimization problem - such as the feasibility problem for the interval case - that has the undominated elements as its solution. So currently we use an approximation approach for dealing with this kind of problem: we discretize the space of optimization vectors and perform pairwise comparisons between points in this grid; this approach is infeasible for problems with non-small $n$.

This case is illustrated in Figure 6 with an example we can solve exactly.

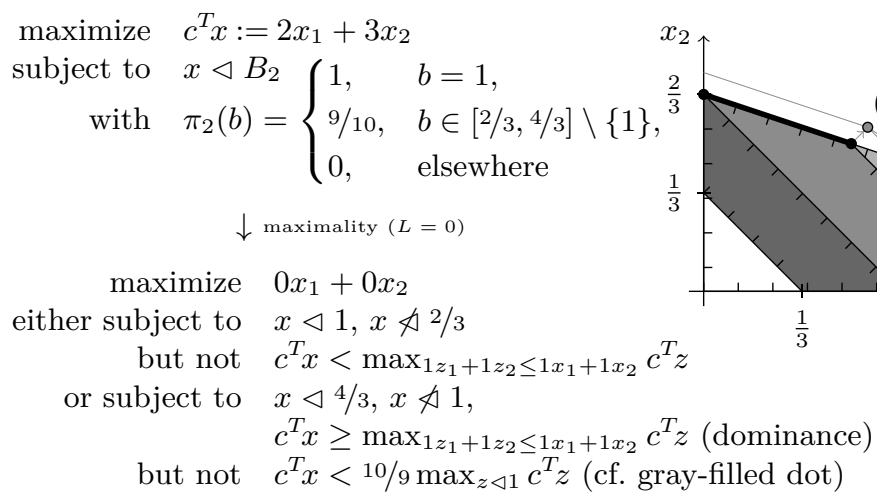

Figure 6. Example linear programming problem with possibilistic uncertainty and maximality - note the modified possibility distribution: the inner feasible set is shaded dark gray, (the infeasible part of) the 1-outer feasible set is shaded gray, the $9 / 10$-outer feasible set is shaded light gray; the solution set of is drawn using thick black lines and the hatched area again consists of optimization vectors that are nonmaximal because they are dominated (cf. Figure 4); the solution set consists of a union of intersections of convex sets of optimization vectors. 


\section{Conclusions}

We have used simple two-valued utility functions. Our approach is in principle compatible with more complex utility functions. For example, in this paper, we only deal with uncertainty in the constraints. Uncertainty in the objective function could in principle be taken into account directly in the utility function of the decision problem. To keep the computational complexity of the problems we study still somewhat manageable, we have refrained from considering more complex utility functions - but it is an obvious avenue for further research.

Currently, the two main new contributions of our research, as presented in this paper, are the formulation of the feasibility problem for the intervalmaximality case and the formulation of the efficiently solvable nested optimization problem for the possibility-maximinity case. We have bumped our head discovering that finding an efficient approach for determining dominance is a worthy goal. But this should not divert all our attention away from adding other types of uncertainty models - such as linear-vacuous previsions, the next step up in complexity - to the list of those we can efficiently find maximin solutions for.

Acknowledgments. This research was supported by the IWT SBO project 60043, "Fuzzy Finite Element Method". We thank the reviewers for comments that were useful and to the point.

\section{References}

1. Ben-Tal, A., Nemirovski, A.: Robust optimization - methodology and applications. Mathematical Programming 92(3), 453-480 (2002)

2. Bertsimas, D., Tsitsiklis, J.N.: Introduction to linear optimization. Athena Scientific (1997)

3. Charnes, A., Cooper, W.W.: Chance-constrained programming. Management Science 6(1), 73-79 (1959)

4. Dantzig, G.B.: Linear programming under uncertainty. Management Science 1(3/4), 197-206 (1955)

5. Fiedler, M., Nedoma, J., Ramík, J., Rohn, J., Zimmermann, K.: Linear Optimization Problems with Inexact Data. Springer (2006)

6. Jamison, K.D., Lodwick, W.A.: Fuzzy linear programming using a penalty method. Fuzzy Sets and Systems 119(1), 97-110 (2001)

7. Quaeghebeur, E., Shariatmadar, K., De Cooman, G.: Constrained optimization problems under uncertainty with coherent lower previsions. Fuzzy Sets and Systems (in press)

8. Sahinidis, N.V.: Optimization under uncertainty: state-of-the-art and opportunities. Computers \& Chemical Engineering 28(6-7), 971-983 (2004)

9. Soyster, A.L.: Convex programming with set-inclusive constraints and applications to inexact linear programming. Operations Research 21(5), 1154-1157 (1973)

10. Troffaes, M.C.M.: Decision making under uncertainty using imprecise probabilities. International Journal of Approximate Reasoning 45(1), 17-29 (2007)

11. Walley, P.: Statistical Reasoning with Imprecise Probabilities, Monographs on Statistics and Applied Probability, vol. 42. Chapman \& Hall, London (1991) 